\title{
Cultura Cultura
}

Revista de Historia Teoria das ldeaias - Revista de História e Teoria das Ideias

Vol. 32 | 2013

O surgimento da ciência moderna na Europa

\section{Dinamismo inconsciente en Leibniz}

Unconscious dynamism in Leibniz

\section{Leticia Cabañas}

\section{OpenEdition \\ Journals}

\section{Edición electrónica}

URL: http://journals.openedition.org/cultura/2016

DOI: 10.4000/cultura.2016

ISSN: 2183-2021

\section{Editor}

Centro de História da Cultura

\section{Edición impresa}

Fecha de publicación: 2 diciembre 2013

Paginación: 167-175

ISSN: 0870-4546

Referencia electrónica

Leticia Cabañas, « Dinamismo inconsciente en Leibniz », Cultura [Online], Vol. 32 | 2013, posto online no dia 10 fevereiro 2015, consultado a 19 abril 2019. URL : http://journals.openedition.org/ cultura/2016 ; DOI : 10.4000/cultura.2016

Este documento fue generado automáticamente el 19 abril 2019.

(c) CHAM - Centro de Humanidades / Centre for the Humanities 


\title{
Dinamismo inconsciente en Leibniz
}

\author{
Unconscious dynamism in Leibniz
}

\author{
Leticia Cabañas
}

1 Leibniz es el primer filósofo en lanzar el tema del fondo inconsciente del alma. El rigor en pensar la vida psíquica le llevó a disociar la percepción de la conciencia, rompiendo con la tradición filosófica carente de la idea de una vida psíquica inconsciente e introduciendo dicha noción en su teoría del conocimiento. ${ }^{1}$ Hay pues una psicología en Leibniz en la que desarrolla una pionera teoría del inconsciente, posteriormente muy ligada a la tradición del romanticismo alemán. El tema del inconsciente contaba por tanto ya antes de Freud con una importante tradición teórica. Sin embargo la concepción del inconsciente leibniziana no tiene absolutamente nada que ver con la de Freud. Lo que sí conocía admirablemente Freud era la escuela de Schopenhauer, orientadora del inconsciente en el sentido de un conflicto de la voluntad, pero no es ésta la corriente leibniziana. La novedad en el creador del psicoanálisis es que concibe el inconsciente en una relación de conflicto con la conciencia, como una fuerza que se le opone. Mientras que el método que seguirá Leibniz es radicalmente distinto, basado en analogías estructurales y en los modos de conexión que resuelven las contradicciones. La estrategia empleada en su metafísica madura es la armonización - y no la subordinación - de la experiencia y la teoría, la interacción entre razón y sentimiento.

2 Leibniz desarrolla un punto de vista muy holístico en el tema del conocimiento, respecto a la totalidad de funciones, imaginativas y racionales, de la mente humana. A diferencia de Kant que establece una discontinuidad, un corte radical entre sensibilidad y razón, pretende intelectualizar la experiencia, establecer una diferencia gradual y no sustancial entre el pensamiento y la percepción. ${ }^{2}$ La epistemología leibniziana combina la experiencia sensible y la razón, considerando el espíritu y la materia como los extremos de un continuo. Con ello intenta unir conceptualmente lo que más tarde Kant dividirá entre cosa en sí y apariencia. Efectivamente, no hay en Leibniz una diferencia fundamental entre la percepción natural y el conocimiento intelectual, en cuanto que comparten una misma estructura básica. La estricta distinción entre conocimiento empírico y racional desaparece, no tiene límites fijos. En este tema del conocimiento resulta idóneo aplicar la 
imagen del océano continuo, sin límites, una de las más memorables metáforas concebidas por Leibniz. ${ }^{3}$

Hobbes había buscado desarrollar un modelo sistemático de pensamiento a base de llevar el mecanicismo al estudio científico de la mente. El descubrimiento por Leibniz de la epistemología y de la lógica hobbesiana fue factor importante para reconocer la importancia del conocimiento sensorial. Por influencia suya, el concepto de sentir, de tener percepciones o sensaciones, Leibniz lo interpreta en el sentido amplio de conocer. ${ }^{4}$ La percepción sensorial queda así incluida en el proceso de conocimiento.

4 Los pensamientos están siempre acompañados de sensaciones. ${ }^{5}$ Habría incluso que considerar el pensamiento como una forma especial de percepción, admitir que la ratio tiene una base empírica. La doctrina de la omnisciencia confusa de las mentes no es tanto una teoría del conocimiento, de lo pensado, cuanto una teoría de la sensación, de lo que sentimos. ${ }^{6} \mathrm{Si}$ nos viéramos privados de la experiencia sensible, el punto inicial de todo conocimiento, no tendríamos la posibilidad de desarrollar un pensamiento. ${ }^{7}$ Las estructuras innatas de nuestra mente permanecerían en el estado de simple potencialidad, privadas del elemento material al que aplicarse.

5 Como también es verdad que no tenemos percepciones puras, aisladas de la red de ideas que nos son innatas. Pues los objetos de nuestra experiencia son construcciones mentales que derivan su carácter unitario de la actividad de nuestro intelecto. Sólo si acudimos a las verdades de las ciencias demostrativas podremos introducir un orden en los fenómenos y juzgar acerca de la verdad de las cosas sensibles. Si se quiere proceder demostrativamente hay de algún modo que trascender la experiencia para que las sensaciones sean guiadas por la teoría. ${ }^{8}$

6 Lo que quería Leibniz era salvar la unión del conocimiento teórico y sensorial, establecer una nueva forma de mediación que conectase los procedimientos estrictamente deductivos con el material empírico. En definitiva, tanto el cuerpo como las facultades intelectuales cooperan para producir sentido, no es posible captar el mundo sin el filtro de nuestros conceptos y percepciones. Frente al supuesto intelectualismo atribuido a Leibniz, en su pensamiento se produce una continuidad entre el sentir y la reflexión, lo sensible y lo consciente, lo confuso y lo distinto. Lo racional está ya en germen en lo sensible, mientras que lo sensible se ve penetrado de inteligencia. Tenía razón William James cuando dice : "Leibnitz was a rationalist mind, with infinitely more interest in facts than most rationalist minds can show". ${ }^{9}$ Se rompe el viejo prejuicio sobre el racionalismo por no tener en cuenta la importancia de la percepción sensorial y de la experiencia. Lo que está haciendo Leibniz es abrir una tercera vía entre el apriorismo cartesiano y el empirismo inglés.

7 La visión de Descartes de que el yo no es otra cosa que “...res cogitans, id est, mens, sive animus, sive intellectus, sive ratio..."10 es también el punto de partida de Leibniz, aunque no así el de Spinoza, que comienza por Dios y no por el cogito. Tanto Descartes como Leibniz se sitúan aquí frente al filósofo holandés, que no admite individuos pensantes independientes de la sustancia pensante divina. Pero, a diferencia de Leibniz, para Descartes no hay más que percepciones claras y distintas, no existe una percepción oscura. De acuerdo con su maestro, los cartesianos conciben la percepción unida a los poderes de la razón, por lo que no pueden tomar en consideración las petites perceptions inconscientes leibnizianas. Leibniz no dejará de criticarlos: “...les Cartesiens ont fort manqué, ayant compté pour rien les perceptions dont on ne s'apperçoit pas". ${ }^{11}$ Está de 
acuerdo con Descartes en que el espíritu es siempre activo, pero difiere de él al afirmar que nuestros pensamientos son a menudo inconscientes. ${ }^{12}$

Ateniéndonos a la actividad perceptiva, a los estados internos de las sustancias, la diferenciación entre percepciones confusas y distintas será fundamental para el sistema de Leibniz. Lo inconsciente, las percepciones confusas, son la base del conocimiento claro y distinto. Es ésta una doctrina profundamente anticartesiana, pues Descartes defendía una mente transparente, en la que no hay nada de lo que no seamos conscientes. ${ }^{13}$ Leibniz cuestionará el rígido marco de la teoría del conocimiento cartesiana, al afirmar que el conocimiento claro no siempre es distinto, a no ser que un análisis de los componentes proporcione sus elementos distintivos. No hay que asimilar lo claro y lo distinto, pues la idea clara puede ser confusa.

9 Tampoco hay para Locke percepciones oscuras $y$, en consecuencia, un lugar para las percepciones inconscientes. Frente a ello, en el Prefacio de los Nuevos Ensayos conecta Leibniz expresamente con la teoría de las petites perceptions ${ }^{14} \mathrm{y}$ adscribe un importante papel psicológico a esas pequeñas percepciones que representan objetos fuera del alcance de la conciencia. Tenemos innumerables percepciones de las que no somos conscientes, contenidos de conciencia no reflexivos. En esa asimetría que se produce entre la razón y la conciencia, multitud de percepciones imperceptibles y las inclinaciones que están a su base permanecen inconscientes. ${ }^{15}$

Para Leibniz, al contrario de Descartes y Locke, hay muchos pensamientos de los que no somos conscientes. Somos omniscientes, pero de forma confusa. Recibimos impresiones de los cuerpos circundantes en forma de pequeñas percepciones. A través de la infinitud de percepciones conectamos con el entero universo. La representación no es meramente intelectual, pues muchas percepciones confusas escapan al control del sujeto. Emergen de una suma de petites perceptions no separadamente discernibles. Nuestro conocimiento no se limita por tanto a la "apercepción" o percepción consciente, sino que alcanza una mayor profundidad. La función de la mónada espiritual consistirá en traducir a un nivel diferente, el de la razón, el entero contenido que posee oscuramente.

11 A pesar de las múltiples críticas que Leibniz dirigió a Spinoza, ejerció sobre él una gran fascinación. Un ejemplo es el interés admirativo que sintió por su psicología, que ya admite la posibilidad de un psiquismo inconsciente. Para Spinoza el deseo es la esencia del hombre, sin embargo aun siendo conscientes de nuestros deseos ignoramos las causas que los determinan. No llegará hasta sugerir - lo que sí hará Leibniz en cierta medida que exista una segunda mente misteriosa sepultada bajo la consciente y dotada de una voluntad y deseos propios. Precisamente donde Spinoza y Leibniz se separan es en el análisis del contenido interno de la racionalidad.

12 La doctrina de las pequeñas percepciones le es del todo extraña a Spinoza. Pero según Leibniz son estas petites perceptions en el inconsciente del yo las que desde el plano de lo confuso permiten la continuidad hacia las percepciones distintas conscientes. ${ }^{16}$ Nuestra alma se ve atravesada permanentemente por una infinidad de pequeñas percepciones que caen fuera de la luz de la conciencia, pero que acumulándose y sumándose entre sí logran entrar en la esfera consciente. Estas impresiones son todas percibidas, aunque no lleguen a llamar la atención individualmente. Como la percepción del ruido del mar se compone de la percepción de todas las gotas de agua, así las percepciones conscientes bañan en un flujo de pequeñas percepciones inconscientes. Desarrolla Leibniz una teoría puntillista de la sensación, un hormigueo incesante de innumerables percepciones individuales imperceptibles que componen toda percepción consciente. ${ }^{17}$ La percepción, el estado 
interno de la mónada que representa las cosas externas - un concepto clave en su pensamiento - es sólo simple en apariencia. ${ }^{18}$

Reconoce Leibniz que ni la percepción ni la reflexión son posibles sin las pequeñas percepciones. Una progresión continua conduce desde las petites perceptions subconscientes y los apetitos insensibles, pasando por las percepciones y apeticiones distintas, hasta llegar a las operaciones y elecciones conscientes, reflexivas y racionales. Es un transitar sin término fijo de lo confuso a lo distinto, de lo inconsciente a lo consciente, teniendo en cuenta que las sustancias reproducen y encierran en sí la infinitud del universo. ${ }^{19}$ Esta transición gradual entre los diferentes ámbitos muestra la aplicación del principio del orden general - principium quoddam generale -, la lex continui que ya desde sus primeros escritos había considerado Leibniz de una gran utilidad por su valor heurístico de amplio espectro. La inclinación metafísica en Leibniz por la unidad y la homogeneidad se identifica con el principio de continuidad que permite tratar la heterogeneidad.

Lanza Leibniz un puente desde las inclinaciones, impulsos y percepciones insensibles hacia un cálculo formal consciente, en un transcurrir del conocimiento confuso al distinto. Pues el instinto no representa la antítesis de la lumière naturelle, a pesar de operar en la esfera de lo inconsciente. Constituye una ayuda para la razón, anticipando confusamente la capacidad de apercepción, los actos reflexivos del intelecto. No es una simple correspondencia, sino un nexo intrínseco lo que opera entre el instinto y la razón. De acuerdo con la acción central de la lex continui, una transición gradual se genera entre los diversos ámbitos. Pero no se trata de un proceso anímico mecánico, sino de una conexión entre causa efficiens y causa finalis, es decir, una causalidad impulsada hacia un fin.

15 En cuanto al inconsciente leibniziano, está muy ligado al análisis infinitesimal. Como sucede con la curva matemática, la vida psíquica en Leibniz queda sometida a la ley de composición del continuo. Al igual que hay diferenciales de la curva, hay también diferenciales de la conciencia. En su caso el inconsciente se define precisamente como el conjunto de las diferenciales de la conciencia. La apercepción consciente está en Leibniz compuesta por una infinidad de pequeñas percepciones inconscientes o percepciones infinitamente pequeñas. Esas petites perceptions, las diferenciales de la percepción consciente, forman una serie de ordenadas, una serie regular, en la que la integral del cálculo opera una integración, una especie de totalización.

Introduce Leibniz en los Nuevos Ensayos su concepto de apperception para marcar el paso desde las petites perceptions de las que no somos conscientes a la percepción de lo consciente. ${ }^{20} \mathrm{La}$ apercepción es el conocimiento reflexivo del estado interno de la mónada. La diferencia entre percepción y apercepción no implica una discontinuidad, sino por el contrario, se establece una unión entre sensibilidad y razón de acuerdo con el principio conductor de la continuidad respetado en todo lugar que niega el vacío y los límites entre las entidades. ${ }^{21}$ La sucesión de estados internos de la mónada está regulada, de modo que cada estado es continuación natural del que le precede, en una serie causal de estados mentales que se sucede al infinito. ${ }^{22}$ Un proceso continuo de desarrollo que se extiende más allá del límite de la conciencia y que entronca con la idea barroca de la luz, por oposición a la visión clásica: no hay claridad que no se obtenga de un fondo oscuro.

17 Nos dice Leibniz que las infinitas percepciones inconscientes constituyen al individuo, juegan un papel crucial en la individuación de las sustancias. ${ }^{23} \mathrm{~A}$ su vez el yo, como principio unificante, asegura la unidad de las percepciones. La multiplicidad se proyecta 
en la unidad del sujeto, siguiendo una típica tendencia operativa del pensar leibniziano que es la integración de lo múltiple en lo uno, herencia de la máxima unum ex omnibus, omnia ex uno del jesuita Athanasius Kircher. ${ }^{24}$

18 La acción racional incorpora el nivel apetitivo del sujeto. Una tesis central en la monadología de Leibniz es que todos los seres están dotados de percepción y apetito, modificaciones internas en constante lucha por alcanzar un conocimiento cada vez más preciso, el paso de las percepciones oscuras a las percepciones claras. Las mentes contienen un principio de acción, la fuerza vital que anima a la mónada y que causa las variadas sensaciones que la atraviesan, de acuerdo con una ley serial que desarrolla espontáneamente. Las mónadas son espontáneas, producen sus propios estados y no están determinadas por ninguna causa exterior. Todo lo que le sucede a la sustancia está ya contenido en su propia naturaleza dinámica. Leibniz niega el influxus de una sustancia sobre otra, a pesar de que cada una de ellas está virtualmente presente en el resto de mónadas.

Los cambios en la sucesión de percepciones no son arbitrarios, sino que se basan en un "principe interne" o ley rectora de la mónada misma. Esto excluye toda influencia externa y explica la famosa metáfora de la mónada sin ventanas. Las percepciones son emanaciones de la mónada que no recibe nada de fuera y todo lo saca de su propio interior gracias a su naturaleza espontánea. ${ }^{25} \mathrm{La}$ conciencia es una forma de acción o "actio in se ipsum". ${ }^{26}$ La mens es res agens, una sustancia siempre activa que no puede dejar de percibir y donde un pensamiento es causa de nuevos pensamientos. ${ }^{27}$ Las percepciones tienen apeticiones - appetition, appetitus -, es decir, poderes activos, principio del cambio para generar estados subsiguientes, tendencia que determina la transición de una percepción a otra. ${ }^{28}$ Cada percepción depende funcionalmente de las anteriores percepciones y apeticiones de la mónada. ${ }^{29}$

Introduce aquí Leibniz una nueva interpretación de la actividad mental como una forma de movimiento ${ }^{30}$ por impulsos o conatus, ${ }^{31}$ noción que toma de Hobbes y que reformula como fuerza originaria. Las pequeñas percepciones adquieren así un aspecto dinámico, como sensaciones infinitesimales productoras de apeticiones imperceptibles. Con su visión de una percepción activa creadora y no sólo representativa, confiere Leibniz un nuevo significado al uso pasivo de los conceptos tradicionales de sentiri y percipi. Desarrolla entonces un nuevo concepto de experiencia basado en el carácter constructivo-teórico de la experimentación, muy superior al concepto de experiencia de Locke y otros empiristas, que se reduce a la mera suma de las percepciones recibidas a través de los sentidos.

\section{NOTAS}

1. “...nostre ame... elle garde tousjours en sa nature les traces de tous ses estats precedans avec un souvenir virtuel...", A Arnauld, 14 julio 1686, GP II, 57. Véase traducción en: G.W. Leibniz. Obras filosóficas y científicas, vol. 14, Comares, Granada, 2007 ss., p. 48-49. En lo sucesivo OFC, seguido de volumen y página. 
2. “...il y a des degrés en toutes choses”, Considerations sur la doctrine d'un Esprit Universel Unique, GP VI, 537.

3. “...d'autres comparent le corps entier de nos connoissances à un Ocean qui est tout d'une piece...", NE IV, 21, 4, A VI, 6, 523.

4. "Re recte expensa, tantum illud certum est nos sentire, et sentire congruenter, et quandam in sentiendo observari a nobis regulam", De veritatibus, de mente, de Deo, de universo, A VI, 3, 511.

5. “...nous ne sommes jamais sans pensées et aussi jamais sans sensation... », NE II, 1, 23, A VI, 6, 119.

6. “...quamlibet Mentem simul percipere quicquid fit in toto mundo...”, De plenitude mundi, A VI, 3 , 524. Cf. : "Chaque Ame connoit l'infini, connoit tout, mais confusement..." PNG §13, GP VI, 604. OFC 2,349; "On pourroit connoître la beauté de l'univers dans chaque ame, si l'on pouvoit deplier tous ses replis...", Ibid.

7. "Les sens nous fournissent la matiere aux reflexions et nous ne penserions pas même à la pensée, si nous ne pensions à quelque autre chose, c'est a dire aux particularités que les sens fournissent", NE II, 21, 73, A VI, 6, 212.

8. Massimo MUGNAI, Introduzione alla filosofia di Leibniz, Einaudi, Milán, 2001, pp. 73-76. Cf. “Je demeure cependant d'accord, que... les Sens externes nous sont necessaires pour penser, et que, si nous n'en avions eu aucun, nous ne penserions pas... Les sens nous fournissent de la matiere pour le raisonnement, et nous n'en avons jamais des pensées si abstraites, que quelque chose de sensible ne s'y mêle; mais le raisonnement demande encor autre chose que ce qui est sensible", Lettre touchant ce qui est independant des Sens et de la Matiere, GP VI, 506; “...la liaison des phenomenes, qui garantit les verités de fait à l'egard des choses sensibles hors de nous, se verifie par le moyen des verités de raison...", NE IV, 2, 14, A VI, 6, 374-75.

9. William JAMES, Pragmatism, Echo Library, Middlesex, 2009, p. 15.

10. Meditationes II, AT VII, 27.

11. Mon. §14, GP VI, 608-9. OFC 2, 329.

12. NE II, $19,4$.

13. AT VII, 107.

14. NE, Préface, A VI, 6, 55-57.

15. “...il y a une grande multitude de petites perceptions, où il n'y a rien de distingué...” Mon. \$21, GP VI, 610. OFC 2, 330. Cf.: “...les pensées confuses ne sont autre chose qu'une multitude de pensées qui sont en elles mêmes comme les distinctes, mais qui sont si petites que chacune à part n'excite pas nostre attention et ne se fait point distinguer", Addition à l'Explication du systeme nouveau..., GP IV, 574.

16. “...les perceptions insensibles sont d'un aussi grand usage dans la Pneumatique, que les corpuscules dans la physique; et il est également déraisonnable de rejetter les uns que les autres, sous pretexte qu'elles sont hors de la portée de nos sens", NE Préface, A VI, 6, 56.

17. “...ce qui est remarquable doit estre composé de parties qui ne le sont pas...", NE II, 1, 18, A VI, 6, 117. Cf.: NE II, 9, 4, A VI, 6, 134.

18. "La Perception qui est l'état interieur de la Monade representant les choses externes", PNG $\S 4$, GP VI, 600. OFC 2, 345; Percepción “...état passager, qui enveloppe et represente une multitude dans l'unité...”, Mon. §14, GP II, 311. OFC 2, 329; GP III, 581, GP VI, 608; GP VII, 566 ; “Perceptio est expressio multorum in vere uno seu in substantia simplice", A VI, 1, 286, 20; "Sed perceptio nihil aliud est, quam ille ipsa repraesentatio variationis externa in interna", GP VII, 399, ca. 1706.

19. Antonio LAMARRA, "Notes on Reason and Instinct in the Nouveaux essais", en Leibniz und Europa, VI Congreso Internacional Leibniz, 1995, p. 205.

20. NE II, 9, 4, A VI, 6, 134. Cf. “...l'Apperception qui est la Conscience, ou la connoissance reflexive de cet état interieur...”, PNG §4, GP VI, 600. OFC 2, 345. 
21. "La Loy de la Continuité porte que la Nature ne laisse point de vuide dans l'ordre qu'elle suit...”, NE III, 6, 12, A VI, 6, 307. Cf. “...nullam transitionem fieri per saltum”, A de Volder, 24 marzo-3 abril 1699, GP II, 168.

22. "Omnis cogitatio animum... occupat aliqua cogitandi serie secundum quandam certam legem", De affectibus, 20-22 abril 1679, A VI, 4B, 1424. Cf. "Car chaque perception precedente a de l'influence sur les suivantes, conformement à une loy d'ordre", Eclaircissement des difficultés que Monsieur Bayle a trouvées dans le systeme nouveau de l'union de l'ame et du corps, GP IV 522; "Operatio autem animae propria est perceptio, et unitatem percipientis facit perceptionum nexus, secundum quem sequentes ex pracedentibus derivantur", A des Bosses, GP II, 372. OFC 14, 269; Martin SCHNEIDER, "Leibniz Theorie der Aktion im Jahrzent vor dem Discours de métaphysique. 1677-1686", Studia Leibnitiana, XXXIII/1, 2001, p. 110.

23. "Ces perceptions insensibles marquent encore et constituent le même individu, qui est caracterisé par les traces, qu'elles conservent des estats précedens de cet individu, en faisant la connexion avec son estat present...”, NE Préface, A VI, 6, 55.

24. “...toute multitude suppose des veritables unités...", A la electora Sofía, 12 junio 1700, GP VII, 552. 25. “...naturellement rien ne nous entre dans l'esprit par dehors, et c'est une mauvaise habitude que nous avons, de penser comme si nostre ame recevoit quelques especes messageres et comme si elle avoit des portes et des fenestres”, DM XXVI, A VI, 4B, 1571. OFC 2, 191.

26. “...conscientia, id est actio in se ipsum...”, De affectibus, 20-22 abril 1679, A VI, 4B, 1411.

27. "Cogitatio causa est alterius cogitationis...", Ibid, 1424. Cf. "La substance qui a de la perception, estant naturellement representative de tout l'univers suivant son point de veue, ne sauroit jamais cesser de representer, comme l'univers ne cesse jamais d'agir", Borrador de carta a Hartsoeker, 6 febrero 1711, GP III, 521.

28. "L'action du principe interne, qui fait le changement ou le passage d'une perception à une autre, peut être appellée Appetition... ”, Mon. \$15, GP VI, 609. OFC 2, 329.

29. "L'ame est excitée aux pensées suivantes par son object interne, c'est à dire par les pensées precedentes”, A Jacquelot, 9 febrero 1704, GP III, 464. Cf. Martin SCHNEIDER, "Leibniz über Geist und Machine", Philosophisches Jahrbuch, 92, 1985, p. 342-43.

30. “...comme le mouvement mene la matiere de figure en figure, l'appetit mene l'ame d'image en image...", A la reina Sofía Carlota, 8 mayo 1704, GP III, 347.

31. “... appetitus, seu agendi conatus ad novam perceptionem tendens”, GP VII, 330.

\section{RESÚMENES}

El rigor en pensar la vida psíquica le lleva a Leibniz a disociar la percepción de la conciencia, rompiendo con la tradición filosófica, carente de la idea de una vida psíquica inconsciente. Nuestro conocimiento no se limita a la "apercepción" o percepción consciente, sino que alcanza una mayor profundidad. Desarrolla Leibniz una teoría puntillista de la sensación, un hormigueo incesante de innumerables percepciones individuales imperceptibles componen toda percepción consciente.

By thinking psychic life with accuracy, Leibniz is led to a dissociation of perception and consciousness which breaks away from a philosophical tradition that lacks an idea of unconscious psychic life. Our knowledge is not limited to "apperception" or an unconscious per ception. On the contrary, it reaches a greater depth. Leibniz develops a "pointillist" theory of 
perception: every conscious perception is composed by an unceasing tingle of countless individual perception.

\section{ÍNDICE}

Keywords: consciousness, aperception, perception, theory of sensation, G. W. Leibniz

Palabras claves: conciencia, apercepción, percepción, teoría de la sensación

\section{AUTOR}

\section{LETICIA CABAÑAS}

Madrid

Doctora en Filosofía por la Universidad Complutense de Madrid con la tesis "Lógica y Metafísica en Leibniz: del cálculo lógico a la reforma de la metafísica”. Ha escrito artículos y participado en congresos nacionales e internacionales sobre Leibniz. Es miembro de la Leibniz-Gesellschaft de Hannover y de la Sociedad Española Leibniz.

Leticia Cabañas has written her Dissertation, "Logic and Metaphysics in Leibniz: from logical Calculus to the Reform of Metaphysics", at the Universidad Complutense de Madrid (Spain). She has written many articles and attended national and international congresses on Leibniz. She is member of the German- and the Spanish Leibniz Society (Leibniz-Gesellschaft; Sociedad Española Leibniz). 